\title{
Erster JAK-Inhibitor für PsA zugelassen
}

Mit Tofacitinib wurde der erste Januskinase-Inhibitor für die Therapie der Psoriasis-Arthritis (PsA) zugelassen. Er wirkt effektiv gegen die komplexen Beschwerden der PsA - sowohl bei Patienten ohne bisherige TNFaTherapie als auch bei Patienten mit einer solchen Vortherapie.

Zulassungsrelevant sind die beiden Phase-III-Studien OPAL BROADEN und OPAL BEYOND. Die dreiarmige OPAL-BROADEN-Studie vergleicht Tofacitinib mit Placebo und Adalimumab als aktivem Vergleichspräparat bei Patienten mit PsA, die auf mindestens ein konventionelles synthetisches Basistherapeutikum (csDMARD) nicht ausreichend angesprochen hatten und nicht mit einem TNFa-Hemmer vorbehandelt waren [1]. In der OPAL -BEYOND-Studie wird Tofacitinib $\left(X\right.$ eljanz $\left.{ }^{\circledR}\right)$ placebokontrolliert bei Patienten untersucht, die auf mindestens einen TNFa-Hemmer unzureichend angesprochen hatten [2]. In der ORAL-BROADEN-Studie bei TNFa-Hemmer-naiven Patienten war Tofacitinib Placebo überlegen und ähnlich wirksam wie Adalimumab (primärer Endpunkt: ACR20-Ansprechen: $50 \%$ vs $33 \%$ vs $52 \%$ ). Am Studienende nach zwölf Monaten lagen die ACR20-Ansprechraten bei $68 \%$ unter Tofacitinib und bei $60 \%$ unter Adalimumab.

Auch die Lebensqualität verbesserte sich unter Tofacitinib innerhalb von drei Monaten deutlicher als Placebo und etwa ähnlich wie unter Adalimumab. In der OPEN-BEYOND-Studie mit TNFa-Hemmer vorbehandelten Patienten erreichten $47 \%$ unter Tofacitinib ein ACR20-Ansprechen und profitierten damit ebenso wie TNFaHemmer-naive Patienten. Nach einem TNFa-Blocker lagen die ACR-20-Ansprechraten bei knapp $60 \%$ (Plazebo: $27,7 \%$ ), nach mindestens einem TNFa-Hemmer bei $50 \%$ und nach mindestens zwei TNFa-Hemmern bei 33,3 \% (Placebo: 21,2 \%). „Eine TNFaVortherapie ist kein Nachteil für die Behandlung mit Tofacitinib", betonte
Dr. Frank Behrens, Ärztlicher Direktor und geschäftsführender Gesellschafter des CIRI (Centrum für innovative Diagnostik und Therapie - Rheumatologie/ Immunologie) in Frankfurt.

\section{Auch Enthesitis und Daktylitis wirksam reduziert}

Auch die für die PsA typischen Symptome Enthesitis und Dactylitis wurden von dem JAK-Inhibitor günstig beeinflusst, ebenso die Hauterscheinungen. Tofacitinib wird damit der Komplexität der Symptomatik bei PsA

durchaus gerecht. Und es hat den Vorteil der oralen Applikation.

\section{Literatur \\ 1. Mease P et al (2017) N Engl J Med 377:1537-1550 \\ 2. Gladman D et al (2017) N Engl J Med 377:1525-1536}

rheuma plus $2018 \cdot 17: 193$ https://doi.org/10.1007/s12688-0180223-x

(c) Springer-Verlag GmbH Austria, ein Teil von Springer Nature 2018

\author{
Quelle: Dr. Beate \\ Fessler, Pressege- \\ spräch Pfizer:Tofa- \\ citinib (Xeljanz ${ }^{\circledast}$ ) \\ Update Rheumato- \\ logie: neue Indika- \\ tionen - bewährte \\ Strategien - Perspek- \\ tiven der JAK-Inhibi- \\ tion", anlässlich des \\ DGRh-Kongresses \\ 2018, Mannheim, \\ 20.09.2018
}

Hier steht eine Anzeige.

$$
\text { 算 Springer }
$$

